Original article

\title{
Cognitive functions in young adults with generalized anxiety disorder
}

\author{
Kiara Leonard, Amitai Abramovitch* \\ Department of Psychology, Texas State University, San Marcos 78666, TX, USA
}

\section{A R T I C L E I N F O}

\section{Article history:}

Received 17 September 2018

Received in revised form 26 October 2018

Accepted 29 October 2018

Available online 17 November 2018

\section{Keywords:}

Anxiety

Worry

Neuropsychology

GAD

Executive function

\begin{abstract}
A B S T R A C T
Background: Anxiety and worry are central symptoms of Generalized Anxiety Disorder (GAD) that have been theorized to negatively impact cognitive functions. However, most of the research has focused on threat-related or emotionally-charged stimuli, and a surprisingly small number of investigations examined 'cold' cognitive functions using classic neuropsychological tests. Such investigations are particularly important given that some theoretical models suggest compensatory mechanisms associated with anxiety that in certain circumstances may result in intact performance. The aim of the present study is to assess the neuropsychological profile associated with GAD, using a comprehensive neuropsychological battery.

Methods: A sample of 23 college students meeting criteria for DSM-5 GAD and 20 control participants completed a psychometrically valid comprehensive computerized neuropsychological battery and clinical questionnaires.

Results: The GAD sample presented with significantly elevated symptomatic rates of anxiety, worry, depression and stress. However, no significant differences were found on any neuropsychological outcome measures or domain indexes. Effect sizes were small, some of which favored the GAD sample. Conclusion: Despite substantial psychopathological burden, GAD exhibited intact cognitive functioning. These results support the Cognitive Control Theory of Anxiety, suggesting that elevated primary anxiety may not impact 'cold' cognitive functions in the absence of threat or substantial cognitive load. Given that this is one of the only studies employing a comprehensive neuropsychological battery in GAD, more research is needed in this population to replicate these results and to examine the impact of anxiety on cognitive functions at varying degrees of cognitive load in this population.
\end{abstract}

(C) 2018 Elsevier Masson SAS. All rights reserved.

\section{Introduction}

Generalized Anxiety Disorder (GAD) is a widespread condition with an estimated lifetime prevalence of $3.7 \%$ worldwide [1]. GAD is characterized by chronically elevated anxiety and difficulty controlling worry about a variety of activities and responsibilities, accompanied by significant somatic and cognitive symptoms [2]. Historically, anxiety, and its cognitive aspect - worry, have been associated with reduced performance in several cognitive domains [3-5] predominantly in the context of threat stimuli [6]. Building upon their own Processing Efficiency Theory [7] that illustrates the negative impact of elevated anxiety on cognition in the context of high cognitive load, Eysenck and colleague's Attentional Control Theory [3] suggests that anxiety reduces attentional control functions which, in turn, hinders cognitive

\footnotetext{
* Corresponding author at: Department of Psychology, Texas State University, 601 University Drive, San Marcos, TX, 78666, USA.

E-mail address: abramovitch@txstate.edu (A. Abramovitch).
}

processing efficiency. Importantly, these models emphasize the effect of threat-related stimuli (i.e., affective, or 'hot') in this population and, to a lesser extent, neutral ('cold') stimuli. [8]. In support of Eysenck's model, studies conducted as early as five decades ago have shown that individuals with high anxiety levels performed significantly worse on 'hot' functions than individuals with low levels of anxiety on a modified Stroop task $[9,10]$. Contemporary studies utilizing emotional Stroop tests demonstrate that samples characterized by elevated worrisome thoughts tend to exhibit a bias for the threat-related stimuli, therefore hindering intact cognitive functions [11,12]. Furthermore, multiple studies concluded that threat stimuli in particular, impair attentional control in individuals with high-trait anxiety $[13,14]$. In addition to attentional control, high worry may be associated with deficient task performance on tests assessing response inhibition, set shifting and working memory $[13,15]$.

Considering 'cold' cognitive functions, the Attentional Control Theory outlines two important mechanisms: First, it suggests that in the context of anxiety and worry, working memory and inhibitory functions may be impaired only in tasks of high 
cognitive load. Second, the theory emphasizes that "Theoretically, high-anxious individuals typically use more processing resources than low-anxious ones in achieving a comparable level of performance" ([3] p. 340). Indeed, Eysenck and colleagues echo Easterbrook's theory [16] suggesting that anxiety leads to narrowing of attention which produces enhanced focus on those task stimuli in particular [16]. Surprisingly, however, very little research is available assessing classic 'cold' neuropsychological function in the context of GAD. In fact, it appears that compared to other major disorders, GAD may be one of the least researched conditions in the context of cognitive function. This has been recently echoed by Hallion and colleagues [17] who noted that most of the research on GAD has been conducted in the context of 'hot' stimuli. This is surprising because both contemporary, and decades old models, include some specific hypotheses regarding the association between anxiety and worry and 'cold' neuropsychological function. Notably, these models focus predominantly on inhibition, set shifting and attention functions - for which very little is known in GAD samples. Moreover, very little is known about memory, visuospatial function, processing speed and a number of executive functions in GAD [17].

The limited research available on neuropsychological functions in the context of GAD is characterized by studies employing only a few tests and, to our knowledge, no comprehensive neuropsychological investigation has been published in this population to date. However, the available literature provides preliminary data pertaining to several cognitive domains. In the memory domain, a few studies found intact verbal memory in GAD compared to controls [18-20]. In the domain of non-verbal memory, one study found reduced nonverbal memory in GAD [21] whereas another found intact performance on the same task [20]. Using the Trail Making-A to assess processing speed, one study found no difference between a large control sample and very small GAD sample ( $n=7$ [18]). In the domain of executive functions, one study reported comparable performance on the Trail-Making B set shifting task between a GAD sample and non-clinical controls [18]. Conversely GAD participants were found to perform significantly worse than controls on the Wisconsin Card Sorting Test [21]. Investigations into inhibitory function in GAD yielded comparable performance on a Go/No-Go task but reduced performance on a Stroop task [17]. Anecdotal evidence from studies comparing GAD and control samples indicates comparable performance on the N-Back working memory task, the Block Design visuospatial test, as well as verbal fluency [21,22].

In sum, the field of cognitive function in GAD is characterized by an unusually small body of literature. The current literature is inconsistent in terms of research results but tends to reveal intact performance in GAD in most cognitive domains. This literature has some limitations such as lack of control of potential confounding factors (e.g., severity of depressive symptoms, stress) and, ultimately, lack in studies utilizing a comprehensive neuropsychological battery to assess cognitive function in GAD. Consequently, a number of researchers have been explicitly calling for more research on 'cold' neuropsychological function in GAD, particularly response inhibition [17,21,23]. Notably, lack of research in GAD is not unique to neuropsychological functions. Indeed, it has recently been noted that there has been far less research published on GAD compared to other major disorders (including essential research on underlying psychopathological mechanisms), even though GAD may be the most prevalent anxiety disorder [24]. In fact, the authors suggested that future research should investigate GAD in university settings due to a finding stating that $7 \%$ of undergraduates may meet criteria for GAD - which is nearly double the pervalence in the general population [25]. Moreover, there is a misconception that GAD is associated with less functional disability than other disorders [26], but in reality, GAD has been found to be associated with substantial functional disabilities. For example, one large study estimated that $56 \%$ of individuals diagnosed with GAD may be characterized by a severe degree of disability, exceeding all other anxiety disorders [27]. In addition, given that the prevailing models of cognitive functions and anxiety are unique in that they highlight both underperformance in certain conditions and cognitive domains, but intact (or even elevated) performance in certain circumstances. Thus, is an urgent need to systematically map 'cold' neuropsychological functions in GAD using comprehensive neuropsychological batteries. To fill in this gap in the literature, the present study's aim was to examine neuropsychological function in GAD compared to controls, using a validated computerized neuropsychological battery, addressing relevant clinical and demographic variables.

\section{Methods}

\subsection{Participants}

Participants were recruited from the student population at a large university in the southwest United States. The present study utilized a two-phase recruitment process. In the first stage, the Penn State Worry Questionnaire (PSWQ [28]) was sent via email to the entire undergraduate population. We received responses from 1563 students who completed the PSWQ and agreed to be recontacted. The cutoff score for the GAD group inclusion (PSWQ total score $\geq 69$ ) was determined using an empirically derived cut off that was shown to predict presence of GAD with $68 \%$ accuracy [29]. The PSWQ cutoff score for the control sample was $\leq 40$. Exclusion criteria for the second phase (in-person assessment) included any history of a neurological condition (e.g., brain injury, epilepsy), non-corrected vision or color blindness. Inclusion criteria included basic English proficiency and age between 1865 . Four hundred and ninety-four students met the criteria for inclusion in the second phase (control group $n=257$, and the GAD group, $\mathrm{n}=237$ ). One hundred, and 124 were randomly selected for invitation from the GAD and control groups, respectively. To minimize 'no shows' and cancellations, we used a randomization process to invite 15 participants from each group to the lab each week. Given that the GAD group had a slightly higher positive response rate than the control group, we were required to randomize and send invitations to 20 more participants from the control group. Of the 100 participants invited from the GAD group, 77 did not respond (Initial PSWQ: $M=71.82, S D=3.21$ ) and 23 completed the second phase (Initial PSWQ: $M=72.61, S D=3.95$ ). No significant difference was found on the PSWQ between the 77 participants who did not respond, to the 23 who responded and completed the study $[F(1,98)=0.96, p=0.329]$. Of the 124 participants invited from the control group, 103 did not respond (Initial PSWQ: $M=34.73, S D=6.37$ ) and 21 completed the second phase (Initial PSWQ: $M=32.90, S D=7.82$ ). No significant difference on the PSWQ was found between the 103 participants who did not respond to the 21 who responded and completed the study ( $F$ $(1,122)=1.28, p=0.259)$. Of the 51 participants who responded to the invitation for the second phase, seven participants did not show for testing. In preparation for analyses, we have identified one participant from the control sample for which self-report measures and GAD diagnosis were extremely contradictory. This participant was found to meet criteria for GAD (as part of a GAD semi-structured screening interview; see Procedure and Measures sections), even though the participant scored very low on the PSWQ. In addition, the participant's scores on the DASS-anxiety, the STAI, and the PSWQ were inconsistent and random on both the total score and itemized level. This participant was excluded from all analyses in the present study. Thus, the final total sample was then comprised of 20 participants in the control group and 23 participants in the GAD group. Of the 23 participants in the GAD 
sample, 10 reported never receiving a DSM diagnosis. The remaining 13 participants in the GAD sample self-reported the following lifetime diagnoses conducted by a licensed psychologist/ psychiatrist: Generalized Anxiety Disorder 30\% ( $n=7)$, Depression 26.1\% ( $n=6)$, Social Anxiety Disorder 21.7\% ( $n=5)$, Panic Disorder $13 \%(n=3)$, Eating Disorder 13\% ( $n=3)$, Bipolar Disorder 4.3\% $(n=1)$, Post Traumatic Stress Disorder 4.3\% ( $n=1)$, Obsessive-Compulsive Disorder 4.3\% $(n=1)$ and Agoraphobia 4.3\% ( $n=1)$. Demographic information for the two groups is presented in Table 1 . This study was approved by the University's Institutional Review Board in line with Declaration of Helsinki. All participants signed an informed consent. Participants were compensated $\$ 20$ for their time.

\subsection{Measures}

\subsubsection{Clinical measures}

The MINI International Neuropsychiatric Interview (MINI [30]) is a reliable and validated semi-structured diagnostic interview for DSM-V disorders. The present study utilized only the Generalized Anxiety Disorder module.

The Penn State Worry Questionnaire (PSWQ [28]) is a 16-item scale in which the participant responds to statements related to worry. The participants are asked to indicate how much the statement generally applies to themselves by rating the items on a Likert-type scale ranging between 1 (not at all typical of me) and 5 (very typical of me). The PSWQ demonstrated good reliability in clinical samples (Cronbach's $\alpha=.86$ [31]) and in college students ( $\alpha=.80$ [32];). In the present study the PSWQ demonstrated excellent reliability $(\alpha=.98)$.

Depression Anxiety Stress Scale-21 (DASS-21 [33]) is a self-report questionnaire assessing severity of depression, anxiety and stress symptoms over the past week. From a total of 21 items, seven comprise each of the three constructs. The items are scored on a Likert-type scale from 0 (did not apply to me at all) to 3 (applied to me very much or most of the time). The DASS-21 demonstrated excellent reliability in clinical populations (Cronbach's $\alpha=.96, .89$, and 93 for depression, anxiety, and stress, respectively [34]) and in non-clinical populations $(\alpha=.96, .89$, and 93 for depression, anxiety, and stress, respectively [35,36];). In the present study, excellent reliability was demonstrated on all scales $(\alpha=.91, .88$ and .86 for depression, anxiety and stress, respectively).

The State-Trait Anxiety Inventory (STAI $[37,38]$ ) is a self-report questionnaire divided into two subscales: state anxiety and trait anxiety, each consisting of 20 items. The STAI has demonstrated excellent reliability in clinical samples (Cronbach's $\alpha=.95$ and .93 for state and trait, respectively [39]) and non-clinical samples ( $\alpha=.97$ and .95 for state and trait, respectively [40]). In the present study, excellent internal consistency was demonstrated on both the STAI-State $(\alpha=.94)$ and the STAI-Trait $(\alpha=.95)$.

\subsubsection{Neuropsychological measures}

The NeuroTrax Computerized Neuropsychological Battery is a 45minute computerized battery assessing seven major cognitive domains (i.e., memory, executive function, attention, information processing speed, visuospatial function, verbal function and motor skills). This battery has been validated across both clinical (i.e., medical, neurological and psychiatric) and non-clinical populations (e.g. [41,42]) and has demonstrated good reliability [43]. Raw test scores are compared against age and education norms to produce scaled scores that are similar to the Wechsler intelligence scale distribution (i.e., $M=100, S D=15$ ). In addition, the seven domain scores are averaged to yield a Global Cognitive Score. The battery includes the following subtests:

Verbal memory test- Ten pairs of words are presented followed by a list of four words. The participant is instructed to select the one word that was originally presented. Approximately ten minutes after the four sets, a delayed recognition test is administered.

Non-verbal memory test-The participant is presented with eight simple geometric forms and is required to memorize their orientations. In the recognition test, four options are presented in varying orientations and the participant must select the one that was previously presented. Approximately ten minutes after the four repetitions, a delayed recognition test is administered. This subtest measures memory.

Problem solving task- The participant is presented with geometric puzzles. Each puzzle is incomplete puzzle with only three pieces included. The participant is presented six choices from which one completes the puzzle. Increasingly difficult puzzles are then administered. This subtest assesses executive function.

Stroop test- The Stroop test consists of three phases, each of which presents the participant with colored squares on either side of the screen accompanied by a single word. First, a colored word appears and the participant must select the colored square that matches that of a general word (e.g., cat in red letters). Second, the participant must select the square that matches the colored words' meaning (e.g., red). Finally, the participant is presented with the name of a color, but the word's meaning does not correspond to the color of the word (e.g., "RED" is presented in green). This subtest measures executive function.

Expanded Go/No-Go test- Colored squares are presented. The participant is instructed to click on all squares as quickly as possible, except for the red squares. Throughout this subtest, the participant is presented with an increasing number of red squares and distractor stimuli in shorter intervals. This subtest is designed to measure attention, response speed and response inhibition.

Staged information processing speed- The participant is presented with three levels of information processing load: Single

Table 1

Demographic and clinical characteristics.

\begin{tabular}{|c|c|c|c|c|c|c|c|c|}
\hline & \multicolumn{3}{|l|}{ GAD $(n=23)$} & \multicolumn{3}{|c|}{ Control $(n=20)$} & \multirow[t]{2}{*}{$F(1,41) / X^{2}$} & \multirow[t]{2}{*}{ Sig } \\
\hline & Mean/ N(\%) & SD & Range & Mean/ N(/\%) & SD & Range & & \\
\hline Age (years) & 20.33 & 1.42 & $18-24$ & 20.94 & 3.12 & $18-33$ & 0.732 & 0.397 \\
\hline Education (years) & 13.91 & 1.08 & $13-16$ & 14.40 & 1.23 & $13-17$ & 1.904 & 0.175 \\
\hline GPA & 3.31 & 0.65 & $1.22-3.95$ & 3.41 & 0.44 & $2.5-4.0$ & 0.348 & 0.559 \\
\hline$\%$ Females & $20(87 \%)$ & - & - & $13(65 \%)$ & - & - & 0.148 & 0.089 \\
\hline \% Right Handed & $22(96 \%)$ & - & - & $16(80 \%)$ & - & - & 0.167 & 0.110 \\
\hline PSWQ & 69.57 & 11.15 & $25-80$ & 36.95 & 14.81 & $16-74$ & 67.612 & $<0.001$ \\
\hline DASS-21 Depression & 15.22 & 12.06 & $0-40$ & 4.90 & 6.17 & $0-22$ & 11.905 & 0.001 \\
\hline DASS-21 Anxiety & 18.78 & 10.35 & $0-38$ & 5.50 & 8.26 & $0-34$ & 21.184 & $<0.001$ \\
\hline DASS-21 Stress & 22.52 & 9.78 & $4-40$ & 7.70 & 6.75 & $2-30$ & 32.421 & $<0.001$ \\
\hline STAI State & 49.17 & 9.24 & $31-66$ & 31.50 & 8.27 & $20-59$ & 43.107 & $<0.001$ \\
\hline STAI Trait & 56.22 & 7.10 & $42-71$ & 34.80 & 9.06 & $22-57$ & 73.305 & $<0.001$ \\
\hline
\end{tabular}

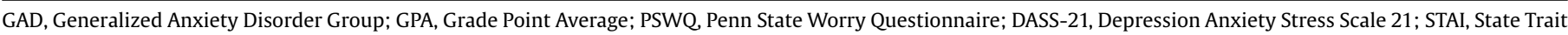
Anxiety Inventory. 
digits, two-digits (e.g., 6-3) and three-digit arithmetic problems (e.g., $3+9-4)$. For each level, the arithmetic problems are presented at three fixed rates and increase in difficulty. The participant is instructed to press the left mouse button if the result is less or equal to 4 and the right mouse button if it is greater than 4 . This subtest measures information processing speed.

Verbal function-The participant is presented pictures of low and high familiarity. The participant is given four word choices, one of which names the object in the picture. In the second portion of this task, the participant must select the word that rhymes with the object, out of four possible options. This subtest assesses verbal function.

Visuospatial processing- The participant is presented with a red pillar in varying 3D locations. The participant is then given four alternative views of the red pillar and is instructed to select the option that corresponds to its vantage point. This subtest assesses visuospatial function.

Catch game- The participant is presented with a small white object falling from the top of the screen and a green bar at the bottom of the screen, which is used to catch the falling white object before it reaches the bottom of the screen. The paddle is maneuvered by utilizing the right and left mouse keys. The mean number of excess moves is a central outcome measure. This subtest measures planning and motor skills.

Finger tapping test- The participant is instructed to click the left mouse button as quickly as possible for $12 \mathrm{~s}$. The participant completes two repetitions of the task. This subtest measures motor skills.

\subsection{Procedure}

Participants from the two pools were randomized each week and invited to the second phase of the study. To increase the validity of neuropsychological data in the present study, participants were asked to refrain from taking stimulants and benzodiazepines or consuming more than two alcoholic beverages within the $24 \mathrm{~h}$ prior to their appointment. This advisory was communicated in both the initial invitation email, as well as the 48-hour reminder email. Upon arrival to the lab, participants signed an informed consent form. Participants were then interviewed using the MINI GAD module individually, administrated by a research assistant who underwent extensive 3 months training on the MINI and neuropsychological testingand had administrated the complete MINI for over a year on a different large research study. Following a careful review and discussion with the first author, the entire GAD sample was confirmed to have met criteria for GAD. Once all participants completed the MINI module interview, they were quietly accompanied to a small computer lab where the computerized portion of the study was administered in a group setting on individual, identical computers. Participants first completed a demographic questionnaire on the computer, which included a detailed self-report of any past diagnoses of a psychological/psychiatric disorder by a licensed psychiatrist or psychologist. Participants then completed the PSWQ DASS-21 and the STAI. Subsequent to the assessments, participants were administered the neuropsychological battery on the same computer which took about $45 \mathrm{~min}$ to complete. The entire session took approximately $75 \mathrm{~min}$.

\subsection{Data analysis}

Data analysis was conducted using SPSS version 24 [44]. Binary nominal variables were analyzed using Pearson's $\chi^{2}$ tests, with Fisher's Exact Test correction. Continuous clinical and demographic variables were analyzed using univariate analysis of variance (ANOVA). Analyses of neuropsychological domain indexes and individual outcome measures were conducted using multivariate analysis of variance (MANOVA). In order to control for familywise inflation of type I error, a priori correction for multiple comparisons was planned using the Bonferroni correction, where 0.05 alpha was divided by the number of outcome measures within a domain or index 'family'.

\section{Results}

\subsection{Demographic characteristics and clinical measures}

Demographic and clinical information are presented in Table 1. Using the MINI GAD module, all participants from the GAD group met DSM-5 criteria for GAD. Both the GAD and control groups consisted primarily of females ( $87 \%$ and $65 \%$ respectively) with no significant difference found between the groups $(p=0.089)$. Additionally, no significant differences were found on age, education, or handedness. As expected, the GAD group scored significantly higher on all clinical self-report measures, including the DASS-21 Depression, the DASS-21 Anxiety, the DASS-21 Stress, the STAI-State, the STAI-Trait and the PSWQ. Mean PSWQ scores in the present study (GAD: $M=69.57, S D=11.15$; control: $M=36.95$, $S D=14.81$ ) were consistent with reported scores of other studies examining GAD participants $[17,31,45]$.

Table 2

Comparisons between the Generalized Anxiety Disorder and Control groups on major neuropsychological domains.

\begin{tabular}{|c|c|c|c|c|c|c|c|}
\hline & \multicolumn{2}{|c|}{ GAD $(n=23)$} & \multicolumn{2}{|c|}{ Control $(n=20)$} & \multirow[t]{2}{*}{$\mathrm{F}(1,41)$} & \multirow[t]{2}{*}{ Sig } & \multirow[t]{2}{*}{ Cohen's $d^{*}$} \\
\hline & Mean & SD & Mean & SD & & & \\
\hline Composite Score & 100.20 & 5.31 & 99.75 & 6.74 & 0.058 & 0.811 & -0.07 \\
\hline Memory & 103.09 & 6.52 & 99.99 & 11.91 & 1.167 & 0.286 & -0.32 \\
\hline Executive Function & 102.36 & 11.04 & 97.57 & 15.33 & 1.409 & 0.242 & -0.36 \\
\hline Attention & 99.54 & 9.34 & 96.00 & 15.21 & 0.869 & 0.357 & -0.28 \\
\hline Information Processing Speed & 93.58 & 8.83 & 95.79 & 8.96 & 0.660 & 0.421 & 0.25 \\
\hline Visuospatial & 102.81 & 12.68 & 107.64 & 8.73 & 2.052 & 0.160 & 0.44 \\
\hline Verbal Function & 97.70 & 10.69 & 98.77 & 7.96 & 0.135 & 0.716 & 0.11 \\
\hline Motor Skills & 102.27 & 8.62 & 102.49 & 9.77 & 0.006 & 0.938 & 0.02 \\
\hline
\end{tabular}

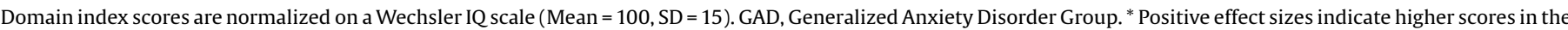
control group, and negative effect size indicate higher scores in the GAD group. 


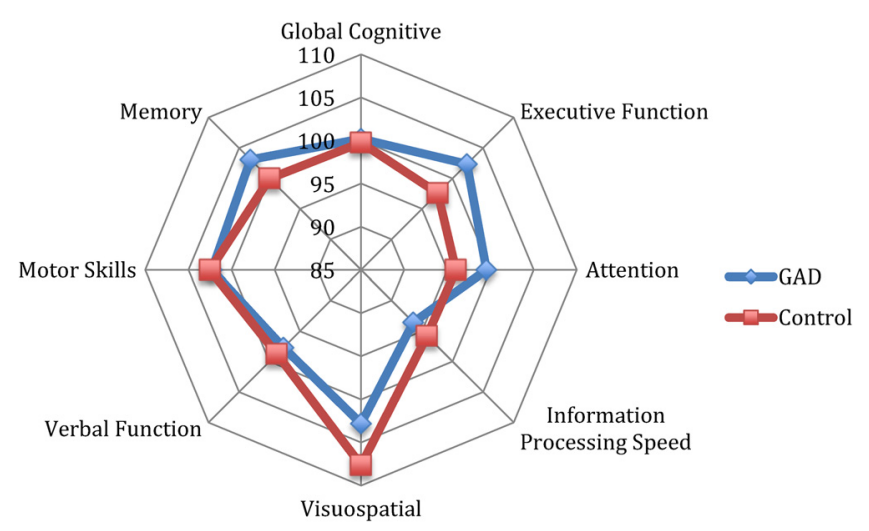

Fig. 1. Comparative Radar Chart of performance index scores across cognitive domains.

\subsection{Neuropsychological functions}

Group comparisons on neuropsychological domain index scores are presented in Table 2. No significant differences were found in any of the major domains ( $p$ 's range 0.160-0.938). All major domains exhibited a small effect size ( $d$ 's range $0.02-0.44$ ). Effect sizes for memory, executive functions and attention favored the GAD sample while processing speed, visuospatial function, verbal functions and motor functions, favored the control group. However, the latter two domains' effects sizes were close to zero. The composite score for overall neuropsychological functioning did not differ between the groups. Moreover, corresponding scaled scores were very close the perfect average population norm (100), with an effect size approaching zero. For graphic representation of the neuropsychological profiles of the GAD and Control groups, see Fig. 1.

No significant difference was found on any domain subsets comprising the main index scores (see Table 3 ) and exhibited a small to medium effect size ( $d$ 's range $0.03-0.60$ ); some favoring the GAD group and some favoring the control. Additionally, there was no difference on the Go/No-Go total commission errors (a primary indicator for response inhibition) between the GAD

Table 3

Comparisons between the Generalized Anxiety Disorder and Control groups on neuropsychological subdomains.

\begin{tabular}{|c|c|c|c|c|c|c|c|}
\hline & \multicolumn{2}{|l|}{$\begin{array}{l}\text { GAD } \\
(n=23)\end{array}$} & \multicolumn{2}{|l|}{$\begin{array}{l}\text { Control } \\
(n=20)\end{array}$} & \multirow[t]{2}{*}{$\mathrm{F}(1,41)$} & \multirow[t]{2}{*}{ Sig } & \multirow[t]{2}{*}{ Cohen's $d^{*}$} \\
\hline & Mean & SD & Mean & SD & & & \\
\hline \multicolumn{8}{|l|}{ Memory } \\
\hline Verbal memory: Total accuracy & 98.01 & 19.16 & 105.96 & 5.57 & 3.200 & 0.081 & 0.56 \\
\hline Delayed verbal memory: Accuracy & 105.35 & 6.70 & 101.51 & 12.76 & 1.583 & 0.216 & -0.38 \\
\hline Non- verbal memory: Total accuracy & 105.76 & 8.37 & 99.35 & 21.61 & 1.732 & 0.195 & -0.39 \\
\hline Delayed non- verbal memory: Accuracy & 103.26 & 7.49 & 93.13 & 22.74 & 4.074 & 0.050 & -0.60 \\
\hline \multicolumn{8}{|l|}{ Executive Function } \\
\hline Expanded Go-NoGo: Composite Score & 98.06 & 19.90 & 99.94 & 21.95 & 0.086 & 0.770 & 0.09 \\
\hline Stroop: Composite score, 3 & 104.98 & 14.46 & 98.31 & 20.28 & 1.573 & 0.217 & -0.38 \\
\hline Catch Game: Total score & 104.04 & 13.74 & 94.47 & 20.05 & 3.412 & 0.072 & -0.56 \\
\hline Problem Solving & 96.89 & 15.17 & 93.57 & 20.24 & 0.377 & 0.543 & -0.19 \\
\hline \multicolumn{8}{|l|}{ Attention } \\
\hline Expanded Go-NoGo: RT & 99.55 & 19.73 & 99.92 & 22.73 & 0.003 & 0.955 & 0.02 \\
\hline Expanded Go-NoGo: RTSD & 98.01 & 19.96 & 94.64 & 22.09 & 0.305 & 0.584 & -0.16 \\
\hline Staged Info Proc Composite 3.3 & 91.81 & 18.19 & 91.65 & 17.64 & 0.001 & 0.978 & -0.01 \\
\hline \multicolumn{8}{|l|}{ Information Processing Speed } \\
\hline Staged info composite score 1.1 & 100.88 & 13.56 & 99.73 & 18.20 & 0.57 & 0.813 & -0.07 \\
\hline Staged info composite score 1.3 & 106.19 & 16.01 & 111.19 & 14.62 & 1.133 & 0.293 & -0.33 \\
\hline Staged info composite score 2.1 & 85.61 & 10.94 & 91.00 & 9.60 & 2.904 & 0.096 & 0.52 \\
\hline Staged info composite score 2.2 & 91.44 & 11.43 & 90.70 & 10.03 & 0.050 & 0.825 & -0.07 \\
\hline \multicolumn{8}{|l|}{ Visuospatial } \\
\hline Visuospatial: Accuracy & 102.81 & 12.68 & 107.64 & 8.73 & 2.052 & 0.160 & 0.44 \\
\hline \multicolumn{8}{|l|}{ Verbal Function } \\
\hline Verbal Function: Rhyming, Accuracy & 99.24 & 11.88 & 97.98 & 11.49 & 0.123 & 0.727 & -0.11 \\
\hline Verbal Function: Matching, Accuracy & 97.70 & 10.69 & 98.77 & 7.96 & 0.135 & 0.716 & 0.11 \\
\hline \multicolumn{8}{|l|}{ Motor Skills } \\
\hline Finger Tapping: Inter- Tap Interval & 97.89 & 11.41 & 99.95 & 17.43 & 0.216 & 0.645 & 0.14 \\
\hline Finger Tapping: Inter- Tap Interval SD & 97.53 & 18.86 & 96.99 & 12.43 & 0.012 & 0.915 & -0.03 \\
\hline Catch Game: Time to first move & 111.38 & 11.43 & 110.51 & 13.70 & 0.051 & 0.822 & -0.07 \\
\hline
\end{tabular}

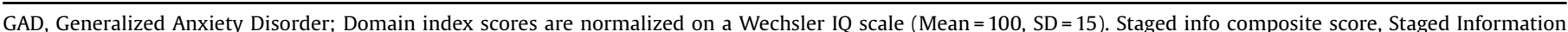
Processing Test Composite Score; * Positive effect sizes indicate higher scores in the Control group, and negative effect size indicate higher scores in the GAD group. 
$(M=93.38, S D=19.72)$ and the control $(M=91.72, S D=20.94)$ groups $(F(1,41)=0.072, p=0.790)$. Mean scaled scores across subsets scores were in the normative performance range (-+ 1 SD). Finally, to assess the effect of clinical variables on group differences, we ran MANCOVAs for the major domains and subdomains, controlling for depressive severity (DASS-21 Depression), state anxiety (STAI-State) and stress (DASS-21 Stress). No changes were found in terms of significant differences, apart from two outcomes: visuospatial functions major domain a $(\mathrm{p}=0.045)$, and verbal memory total score $(\mathrm{p}=0.033)$. However, these two significant results did not survive correction for multiple comparisons.

\section{Discussion}

To our knowledge, this is the first study to utilize a comprehensive neuropsychological battery to assess cognitive functions in a GAD sample; as well as one that is fully computerized. Our results clearly indicate no differences between GAD and controls, with intact performance across cognitive domains in GAD. However, examination of effect sizes reveals an interesting pattern. In terms of major domain effect size directions, the GAD group underperformed on four domains: Information processing speed, motor skills, verbal function and visuospatial functions, with effect sizes ranging between small and null. Yet, effect size directions indicated that the GAD group outperformed the control group on the major domains of memory, executive function, and attention, and had a better overall global performance index with small effect sizes. However, no comparison yielded a significant difference and the largest effect size exemplified a difference of less than half of a standard deviation - an effect size that is not considered a meaningful or clinically relevant cognitive deficiency [46]. Thus, overall comparable and intact performance was found between the GAD and control group.

These results are in line with the limited available research on GAD, where most comparisons across 'cold' neuropsychological outcome measures yield no difference compared to controls [17,18,20-23]. However, these results may be counterintuitive given that the GAD group is characterized by significant psychopathological burden, a factor that has been suggested to be central in terms of its impact on cognitive function [47]. Indeed, the GAD sample in the present study is characterized by moderate to severe levels of anxiety, depression and stress symptoms, as well as extreme intensity of worrisome thoughts. Given that most DSM disorders and subclinical samples exhibit underperformance on cognitive tasks, it may be reasonable to expect to find underperformance in a sample with GAD- a disorder characterized by significant psychopathological burden. However, our results are also in agreement with findings suggesting that anxiety disorders in particular may be associated with smaller effect sizes pertaining to underperformance on cognitive tasks [48-50]. Nevertheless, although Eysenck's model suggests impairment due to anxiety in high cognitive loads, anecdotal evidence also demonstrates underperformance under low cognitive load conditions [51]. However, Eysenck's Attentional Control Theory poses that anxiety may not impair performance when compensatory strategies are utilized [3]. This notion is in line with our findings where participants with GAD in the present study reported substantial psychopathological burden, but did not differ on neuropsychological tasks or GPA suggesting compensatory strategies (i.e., augmented effort or processing resources) may have been employed. However, there is a need for more research in GAD, particularly an assessment of the compensation hypothesis. This line of research is of importance given that it is plausible that a controlled lab environment may inherently reduce the amount of exogenous and endogenous triggers for worrisome thoughts as well as somatic aspects of anxiety, facilitating normative cognitive function.

There are multiple strengths to this study, including being the first study to administer a comprehensive neuropsychological battery in a GAD sample. Moreover, a fully computerized battery was utilized, which minimizes human scoring errors and examiner-examinee interactions, including potential requests for feedback and reassurance and the experiences associated with being directly observed while tested [52]. Nevertheless, the present study is not without limitations. First, sample sizes were relatively small and studies examining larger samples are needed. Indeed, this study may be considered underpowered to detect significant difference with 43 participants and small effect sizes. However, several scholars have pointed out the need to reconsider power conventions in the context of each particular study [53]. In fact, it has been argued that for a novel study that could be considered preliminary (such as the present one), 15-30 participants per group may be sufficient for small effect sizes [54]. In addition, it is particularly important to examine effect sizes and, to a lesser extent, significance, because the former is more informative in the context of neuropsychological test performance in that it can provide information on the presence and degrees of potential deficits and impairments. Second, a formal structured DSM screening was conducted only for GAD and relied on self-report for lifetime formal diagnosis of comorbidity. Third, all participants were college students, which may suggest a certain level of functioning. Nevertheless, there is significant merit in studying GAD among college students given the elevated rates of GAD in this setting and age group [25]. In addition, the GAD group exhibited a symptom severity profile that was similar to data reported on outpatients GAD studies. Notably, nearly half of the GAD sample was defined as non-treatment seekers, which may suggest better psychopathological status. However, our analyses comparing clinical and neuropsychological outcome measures between non-treatment seeking and treatment seeking participants (i.e., participants who reported being diagnosed with a major DSM disorder in the past by a licensed psychologist/psychiatrist) within the GAD sample yielded no significant differences on any clinical measure (all $p$ 's $>0.320$ ), nor on any neuropsychological performance indexes (all $p$ 's $>0.114$ ). Thus, our results indicate that although GAD participants suffer from moderate to severe psychopathological symptoms of depression, stress and anxiety, neither cognitive performance nor GPA are affected by their clinical status or severity. Finally, although contemporary models suggest that working memory may be affected in GAD, the battery used in this study did not include a test of the latter.

\section{Conclusion}

The present study demonstrates that college students meeting criteria for DSM-5 GAD suffer from significant psychopathological burden, but present intact cognitive functions across domains. Although very little research is available regarding 'cold' cognitive functions in GAD, these results are in accord with the predictions of the Cognitive Control Theory of Anxiety, suggesting that individuals suffering from anxiety may be able to present with intact performance on tasks that do not include threatening stimuli or that are not characterized by significant cognitive load. The theory suggests a central role of investment of compensatory effort, which was not assessed in the present study. Future research is needed to replicate and solidify our findings, as well as to attempt to directly assess the specific hypothesis regarding exertion of compensatory effort in GAD. 
Declaration of interest

None.

\section{Funding}

The present study did not receive external funding.

\section{Acknowledgment}

The authors would like to thank Katlyn Brinkley and Estefania Rivas-Bravo for their assistance on this project.

\section{References}

[1] Ruscio A.M., Hallion LS, Lim CCW, Aguilar-Gaxiola S, Al-Hamzawi A, Alonso ], et al. Cross-sectional comparison of the epidemiology of DSM-5 generalized anxiety disorder across the globe. JAMA Psychiatry 2017;74:465-75.

[2] Association, A. P. Diagnostic and statistical manual of mental disorders (DSM5R). American Psychiatric Pub.; 2013.

[3] Eysenck MW, Derakshan N, Santos R, Calvo MG. Anxiety and cognitive performance: attentional control theory. Emotion 2007;7:336-53.

[4] Sarason IG, Sarason BR, Pierce GR. Anxiety, cognitive interference, and performance. J Soc Behav Personality 1990;5:1-18.

[5] Wells A. A cognitive model of generalized anxiety disorder. Behav Modif 1999;23:526-55.

[6] Yiend J. The effects of emotion on attention: a review of attentional processing of emotional information. Cogn Emot 2010;24:3-47.

[7] Eysenck MW, Calvo MG. Anxiety and performance: The processing efficiency theory. Cogn Emot 1992;6:409-34.

[8] Abelson RP. Computer simulation of "hot cognitions". In: Tomkins SS, Messick $\mathrm{S}$, editors. Computer simulation and personality: frontier of psychological theory. . p. 277-98.

[9] Hochman SH. The effects of stress on Stroop color-word performance. Psychonomic Sci 1967;9:475-6.

[10] Pallak MS, Pittman TS, Heller JF, Munson P. The effect of arousal on Stroop color-word task performance. Bull Psychon Soc 1975;6:248-50.

[11] Bar-Haim Y, Lamy D, Pergamin L, Bakermans-Kranenburg MJ, van IMH. Threatrelated attentional bias in anxious and nonanxious individuals: a metaanalytic study. Psychol Bull 2007;133:1-24.

[12] Derakshan N, Eysenck MW. Anxiety, processing efficiency, and cognitive performance. Eur Psychol 2009;14:168-76.

[13] Berggren N, Richards A, Taylor J, Derakshan N. Affective attention under cognitive load: reduced emotional biases but emergent anxiety-related costs to inhibitory control. Front Hum Neurosci 2013;7:188.

[14] Najmi S, Amir N, Frosio KE, Ayers C. The effects of cognitive load on attention control in subclinical anxiety and generalised anxiety disorder. Cognit Emot 2015;29:1210-23.

[15] Darke S. Anxiety and working memory capacity. Cogn Emot 1988;2:145-54.

16] Easterbrook JA. The effect of emotion on cue utilization and the organization of behavior. Psychol Rev 1959;66:183-201.

[17] Hallion LS, Tolin DF, Assaf M, Goethe J, Diefenbach GJ. Cognitive control in generalized anxiety disorder: relation of inhibition impairments to worry and anxiety severity. Cognit Ther Res 2017;41:610-8.

[18] Airaksinen E, Larsson M, Forsell Y. Neuropsychological functions in anxiety disorders in population-based samples: evidence of episodic memory dysfunction. J Psychiatr Res 2005;39:207-14.

[19] Becker ES, Roth WT, Andrich M, Margraf J. Explicit memory in anxiety disorders. J Abnorm Psychol 1999;108:153-63.

[20] Zalewski C, Thompson W, Gottesman II. Comparison of neuropsychologica test performance in PTSD, generalized anxiety disorder, and control Vietnam veterans. Assessment 1994;1:133-42.

[21] Tempesta D, Mazza M, Serroni N, Moschetta FS, Di Giannantonio M, Ferrara M, et al. Neuropsychological functioning in young subjects with generalized anxiety disorder with and without pharmacotherapy. Prog NeuroPsychopharmacol Biol Psychiatry 2013;45:236-41.

[22] Stefanopoulou E, Hirsch CR, Hayes S, Adlam A, Coker S. Are attentional control resources reduced by worry in generalized anxiety disorder? J Abnorm Psychol 2014:123:330-5.

[23] Grillon C, Robinson OJ, O'Connell K, Davis A, Alvarez G, Pine DS, et al. Clinica anxiety promotes excessive response inhibition. Psychol Med 2017;47:484-94.

[24] Newman MG, Przeworski A. The increase in interest in GAD: commentary on asmundson \& asmundson. J Anxiety Disord 2018;56:11-3.

[25] Eisenberg D, Hunt J, Speer N. Mental health in American colleges and universities: variation across student subgroups and across campuses. J Nerv Ment Dis 2013;201:60-7.

[26] Newman MG, Llera SJ, Erickson TM, Przeworski A, Castonguay LG. Worry and generalized anxiety disorder: a review and theoretical synthesis of evidence on nature, etiology, mechanisms, and treatment. Annu Rev Clin Psycho 2013;9:275-97.
[27] Kessler RC, Aguilar-Gaxiola S, Alonso J, Chatterji S, Lee S, Ormel J, et al. The global burden of Mental disorders: an update from the WHO World Mental Health (WMH) surveys. Epidemiol Psichiatr Soc 2009;18:23-33.

[28] Meyer TJ, Miller ML, Metzger RL, Borkovec TD. Development and validation of the Penn State Worry Questionnaire. Behav Res Ther 1990;28:487-95.

[29] Fresco DM, Mennin DS, Heimberg RG, Turk CL. Using the Penn State Worry Questionnaire to identify individuals with generalized anxiety disorder: a receiver operating characteristic analysis. J Behav Ther Exp Psychiatry 2003;34:283-91.

[30] Sheehan DV, Lecrubier Y, Sheehan KH, Amorim P, Janavs J, Weiller E, et al. The mini-international neuropsychiatric interview (M.I.N.I.): The development and validation of a structured diagnostic psychiatric interview for DSM-IV and ICD-10. J Clin Psychiatry 1998;59(Suppl. 20)22-33 quiz 34-57.

[31] Brown TA, Antony MM, Barlow DH. Psychometric properties of the penn State worry questionnaire in a clinical anxiety disorders sample. Behav Res Ther 1992;30:33-7.

[32] Carter MM, Sbrocco T, Miller Jr. O, Suchday S, Lewis EL, Freedman RE. Factor structure, reliability, and validity of the Penn State Worry Questionnaire: differences between African-American and White-American college students. J Anxiety Disord 2005;19:827-43.

[33] Lovibond PF, Lovibond SH. The structure of negative emotional states: comparison of the Depression Anxiety Stress Scales (DASS) with the Beck Depression and Anxiety Inventories. Behav Res Ther 1995;33:335-43.

[34] Brown TA, Chorpita BF, Korotitsch W, Barlow DH. Psychometric properties of the Depression Anxiety Stress Scales (DASS) in clinical samples. Behav Res Ther 1997:35:79-89.

[35] Crawford JR, Henry JD. The Depression Anxiety Stress Scales (DASS): normative data and latent structure in a large non-clinical sample. Br J Clin Psychol 2003:42:111-31.

[36] Sinclair SJ, Siefert CJ, Slavin-Mulford JM, Stein MB, Renna M, Blais MA. Psychometric evaluation and normative data for the depression, anxiety, and stress scales-21 (DASS-21) in a nonclinical sample of U.S. adults. Eval Health Prof 2012;35:259-79.

[37] Marteau TM, Bekker H. The development of a six-item short-form of the state scale of the Spielberger State-Trait Anxiety Inventory (STAI). Br J Clin Psychol 1992;31(Pt 3):301-6.

[38] Spielberger CD, Gorsuch RL, Lushene RE. Manual for the state-trait anxiety inventory. 1970.

[39] Gros DF, Antony MM, Simms LJ, McCabe RE. Psychometric properties of the State-Trait Inventory for Cognitive and Somatic Anxiety (STICSA): comparison to the State-Trait Anxiety Inventory (STAI). Psychol Assess 2007; 19:369-81.

[40] Ortuno-Sierra J, Garcia-Velasco L, Inchausti F, Debbane M, Fonseca-Pedrero E. New approaches on the study of the psychometric properties of the STAI. Actas Españolas de Psiquiatría: Acepsi 2016;44:83-92.

[41] Dwolatzky T, Whitehead V, Doniger GM, Simon ES, Schweiger A, Jaffe D, et al. Validity of a novel computerized cognitive battery for mild cognitive impairment. BMC Geriatrics 2003;3:4.

[42] Schweiger A, Abramovitch A, Doniger GM, Simon ES. A clinical construct validity study of a novel computerized battery for the diagnosis of ADHD in young adults. J Clin Exp Neuropsychol 2007;29:100-11.

[43] Schweiger A, Doniger GM, Dwolatzky T, Jaffe D, Simon ES. Reliability of a novel computerized neuropsychological battery for mild cognitive impairment. Acta Neuropsychol 2003;1:407-13.

[44] IBM. IBM SPSS statistics for windows. 26.0 ed. Armonk, NY: IBM Corp.; 2016.

[45] Behar E, Alcaine O, Zuellig AR, Borkovec TD. Screening for generalized anxiety disorder using the Penn State Worry Questionnaire: A receiver operating characteristic analysis. J Behav Ther Exp Psychiatry 2003;34:25-43.

[46] Abramovitch A, Schweiger A. Misuse of cognitive neuropsychology in psychiatry research: the intoxicating appeal of neo-reductionism. Behavior Therapist 2015;38:

[47] Stordal KI, Mykletun A, Asbjornsen A, Egeland J, Landro NI, Roness A, et al. General psychopathology is more important for executive functioning than diagnosis. Acta Psychiatr Scand 2005;111:22-8.

[48] Castaneda AE, Suvisaari J, Marttunen M, Perala J, Saarni SI, Aalto-Setala T, et al. Cognitive functioning in a population-based sample of young adults with anxiety disorders. Eur Psychiatry 2011;26:346-53.

[49] Lipszyc J, Schachar R. Inhibitory control and psychopathology: a meta-analysis of studies using the stop signal task. J Int Neuropsychol Soc 2010;16:1064-76.

[50] Wright L, Lipszyc J, Dupuis A, Thayapararajah SW, Schachar R. Response inhibition and psychopathology: a meta-analysis of go/no-go task performance. J Abnorm Psychol 2014;123:429-39.

[51] Vytal K, Cornwell B, Arkin N, Grillon C. Describing the interplay between anxiety and cognition: from impaired performance under low cognitive load to reduced anxiety under high load. Psychophysiology 2012;49:842-52.

[52] Woo E. Computerized neuropsychological assessments. CNS Spectrums $2008 ; 13: 14-7$.

[53] Di Stefano J. How much power is enough? Against the development of an arbitrary convention for statistical power calculations. Funct Ecol 2003; 17:707-9.

[54] Whitehead AL, Julious SA, Cooper CL, Campbell MJ. Estimating the sample size for a pilot randomised trial to minimise the overall trial sample size for the external pilot and main trial for a continuous outcome variable. Stat Methods Med Res 2016;25:1057-73. 